\title{
Novel User Centric RFID Fridge Design
}

\author{
Amavi Djimido Noutchet ${ }^{1}$ \\ ${ }^{1}$ Communication Science and Engineering, Fudan University, Shanghai, China \\ Correspondence: Amavi Djimido Noutchet, Communication Science and Engineering, Fudan University, \\ Shanghai, China. E-mail: amnoutchet@gmail.com
}

Received: December 3, $2012 \quad$ Accepted: March 15, $2013 \quad$ Online Published: April 26, 2013
doi:10.5539/cis.v6n2p151
URL: http://dx.doi.org/10.5539/cis.v6n2p151

\begin{abstract}
Radio Frequency Identification technology has been a focal point of recent innovations in home appliance. The smart fridge is now a prominent area of interest for both academics and businesses for its potential to transform our kitchen experience; however the current market price of a smart fridge is highly expensive and out of budget for the average consumer.

A close examination of possible factors shows that while the price of RFID components is constantly decreasing, overall cost remains high enough to make the smart fridge a luxury product.

Implementing adaptive characteristics in high frequency RFID reader/tag will increase the capability to adapt to specific environments such as humidity and different interferences with fridge environment.

In this paper, we introduce a low cost enhanced design smart fridge based on adaptive high frequency RFID combined with the traditional smart fridge features and a freshness detection capability. Our design not only outperforms the existing ones in price but also presents the consumer with information on the freshness of fruits and vegetables.
\end{abstract}

Keywords: RFID, smart fridge, home appliance, food management, health, smart home

\section{Introduction}

Radio Frequency identification technology has been the focal point of innovation for the last two decades. Constant improvement has been made since the days of World War II. Fridges are now equipped with readers and tags of a much smaller size and of increasingly low cost. These improvements have opened the way to integrate RFID technology into brand new applications such as animal breeding, retail management, health conditions tracking devices and various home appliances. Both industry and research have focused on developing smart homes (Chan, Esteve, Escriba, \& Campo, 2008).

Amongst home appliances, the smart fridge has become a vibrant area of recent research, with academics and businesses cooking up ever more innovative concepts. We now have fridges with multimedia capability, entertainment hub possibilities, food management, dietary control and recipe suggestion features.

The RFID systems used while designing the smart fridge come in different flavors from the point of view of the frequency of fridge usage. Selecting the most suitable frequency is subject to the technological developments of systems of different operating frequencies, which is directly related to the cost of system as well as physically to the properties of electromagnetic waves at those different frequencies.

Some of the current RFID fridges available on the market were designed based on Low Frequency (LF), 134kHz. The LF RFID has been in use by RFID systems for years. This fact, together with the less stringent requirements made on the electronics operating in this frequency range, has made this type of RFID very cost effective. Additionally, electromagnetic waves operating in the LF range are the least affected by the presence of metals due to their particular penetration depth. LF waves can also travel through water with minimal attenuation when compared to high frequency waves. These properties make the LF RFID ideal for a fridge environment. However, the LF RFID system does present the major drawback that the reader can only scan one item at any one time. Technical reports and design manual (Amal, 2006) often discuss the characteristics of RFID. This makes using LF RFID technology impractical for our purposes; as one of the main abilities of the smart fridge is the capacity to differentiate itself from the line of sight and single scan method used by bar code technology.

Use has also been made of the Ultra High Frequency (UHF) RFID tag, due to its relatively inexpensive cost. 
UHF systems have established themselves as viable alternatives to the LF and HF system in designing smart fridges. One of the main advantages of UHF RFID is its much faster read rate of transponder information, thus allowing the transmission of higher amounts of data. Despite these encouraging factors, UHF RFID is not very appropriate for the design of the RFID enabled refrigerator. Not only do UHF RFID work well around metals, which reflect UHF electromagnetic waves, but come in very expensive once paired with the necessary tools such as the Universal Software Radio peripheral (USRP), daughterboard and antenna which are necessary for their proper operation (Eric, Nishit, \& Hiran, 2011). In addition, water absorbs UHF waves, making them unusable for animal tagging.

The novel design proposal discussed in this essay presents a concept for a RFID fridge that uses a HF RFID unity. The fridge is to be equipped with an adaptive reader that simultaneously avoids water absorption and metal interference and while providing faster reading rates. It also permits an "all scan together" function, contrary to low frequency RFID unity models. The fridge is capable of recipe suggestion, automatic replenishment, dietary control and in-fridge item management. The user receives updated information of what items are about to expire and what to purchase. The user can also be acknowledged of this information via mobile terminal before a list is generated for online shopping. An order confirmation will enable the fridge to generate and process an online shopping order whereas a denial feedback from the user will allow the fridge to drop the command.

The rest of this paper is organized as follows: Section 2 reviews currently available smart fridge technologies; comparative analysis and proposed fridge architecture is discussed in Section 3, and Section 4 provides a conclusion and future work.

\section{Available Smart Fridge Concepts}

In this section, we enumerate a few of the smart fridge models concepts that are susceptible to hit the market and discuss their scarcity. Numerous designs have been proposed in the last decades; however only fewer of them were made commercial products. This has been highlighted in recent engineering and computer science publications (Suhuai, Hongfeng, Yuan, Jesse, \& Rukshan, 2008).

Although the proposed model has shown its potential in maintaining better nutrition and health, it is still in its preliminary stage. There are many features and areas that can be improved and explored. Possible extensions include Internet connection to allow downloading of recipes in real time, online shopping instruction etc.; the use advanced radio-frequency identification (RFID) tag system to replace bar code system to uniformly store item information; integrate into the fridge a scale to allow automatically weight food or drink such as milk; implement mobile control to allow user to remotely control the fridge using mobile phone.

Another example in academic publication (Philipp, 2011) consists of a midrange RFID reader attached to the fridge door that can read tags for up to $50 \mathrm{~cm}$. The paper resents a concept and prototype for a refrigerator that can visualize the nutritional information by inflating airbags that are built into the fridge. At the same time the airbags function as preventers as they push food out of the fridge if the nutritional data inside the fridge is unhealthy. However the Feedback Fridge at the current stage of development is not aiming to be a real product but to be thought provoking. The author argues that it is doubtful that people would want a product in their households that forces them to a certain behavior and basically denies its proper usage. Even though it might be desirable to have technology shape people's decisions and the effects their actions have.

Fridgets: Digital Refrigerator Magnets, (Jacqueline, Kristy, \& Ryan, 2005) is a great academic achievement that focuses on a need for a human-centered exploration of solutions for the elderly demographic. The design links the elderly person with useful and empowering information and loved ones; entertainment in the form of games and other multimedia possibilities; practical functions, such as shopping lists and medicine reminders are also taken into consideration. Although the central location of the Fridget system is key to creating a presence in the home that will combat loneliness and feelings of isolation, criticism of the design tends to revolve around the mystery of the hardware; the system will be too far expensive.

While most of the designs are still in a prototype phase, fewer of them were made commercial products.

\subsection{Commercial Smart Fridge}

Smart fridge uses RFID technology to lower inventory management cost and protect high value products. According to Joe Pleshek, Chief Executif Officerat Terso Solutions, RFID enables fridge improves regulatory compliance and manage products expiration date. From Terso Solutions to LG and Samsung, companies have expanded the line of RFID enabled enclosures with the new refrigerator that makes better use of space; capture inventory transaction at the item level by the user. 


\subsubsection{Samsung's RF4289 Wi-Fi Smart Fridge}

The RF4289 is a Wi-Fi connected refrigerator with LED and an 8-inch LCD screen with access to kitchen relevant applications such as Google calendar, WeatherBug forecasts, recipes suggestion, AP news and Pandora music. It also allows the user to leave notes for other users. Its retail price is approximately $\$ 3499$.

\subsubsection{LG Smart Fridge for 2012}

The new LG smart fridge is now connected to smart TV and smart phone, which enable convenient monitoring of the operational status of the appliance. Its core features are device-to-device connectivity and enhanced smart management.

\subsubsection{Cool Media Fridge Freezer}

With its 17-inch high definition LCD screen, this fridge boasts superior cooling performance and features. It is highly rated for its energy efficiency, integrated speakers, USB ports, CD/DVD and its high adjustable front supports. The price for the KG28FM50 is approximately Euro 2100.

\subsection{The Current Scarcity of Smart Fridges}

Smart appliances, including RFID fridges, are about to revolutionize how we spend our time at home, especially in regards our kitchen experience. Smart fridge technology involves the integration of many other technologies embracing Radio Frequency Identification technology.

RFID works excellently in a lab environment, but has many high hurdles to overcome before it can completely replace current scanning technology.

\subsubsection{The Issue of Price}

While retailers are already experimenting with RFID tags on high-ticketed, non-grocery items, the technology is still too expensive for many low value items found in grocery stores for instance, potato, tomato and other items. (Brad, Alfonso, \& Raj, 2006) of the RFID lab at University of Wisconsin, agrees that cost is still a problem, since for most organizations; the main barrier to adoption today is lack of Return On Investment. To overcome this, tag costs must come down further, and there must be some very visible examples of companies realizing a significant return on investment across multiple cases.

Ongoing research is developing promising solutions; one promising front is conductive ink and the emerging field of "printable tags".

\subsubsection{The Issue of Maturity}

Currently, RFID programs are in the infant stages of their development with the majority of applications being centered around distribution centers, where pallets or truckloads are scanned at one time by a reader picking up an RFID tag on the outside of each pallet. While a few individual products are equipped with these tags (mostly high-end electronics or building materials), the size and expense of the current crop of tags is prohibitive for most single-item use. So, the application of RFID technology to the smart fridge is still mostly prototyped in a lab environment but the feasible technology is imminent. The technology has been covered by academics papers where practitioners where constraint to swich from the ideal to a more convenient choice, (James, Greggory, Michael, \& Bret, 2007) in order to reach their research goal.

\subsubsection{Issue of Privacy}

Besides cost and lack of stability, privacy issues are a growing concern. While the use of RFID increases security in some areas, the very nature of RFID communication poses a number of new privacy and security risks. More and more consumer groups are protesting, saying that consumer privacy will be threatened. RFID security has even come up for discussion in several US states. One of the most vocal consumer groups against the implementation of RFID in retail stores is (Consumers Against Supermarket Privacy Invasion and Numbering [CASPIAN], 2013)" headed by Katherine Albrecht. Albrecht states two examples, one of which is hypothetical, where consumer privacy is abused.

\subsubsection{Issue of Security}

The issues relating to security are often those that involve fake or illegitimate tags, rather than readers. The main security problem is that tags can very easily be counterfeited or copied. The smart Fridge is frequently designed to perform online shopping and communication functions, opening the door to Internet traffic, known for its security issues. This gives rise to a host of problems. Given the example of tagged objects and the intelligent fridge that warns its users if a product has exceeded its best-before-date, the question arises how this date will be protected against alterations on the way of the product throughout the supply chain, as well as who is responsible 
for the accuracy of this data.

Imagine receiving spam email, advertisement from your retailers or your Smart Fridge telling you that your fridge is empty when you had just replenished the day before.

Another concern of security and privacy issue is directly related to EPC architecture itself. As in the case of RFID wireless communication, true security is sorely lacking in the design of the EPC global Network and especially the ONS. It is likely that the final design of the EPC global Network will include some kind of central certificate authority (CA), (Traub, 2005). How scalable this will turn out in real applications, remains unclear. Moreover, such a CA would constitute another single point of failure and would have to be trusted by all current and future parties involved. The forthcoming EPCIS Discovery Services should be designed carefully, as these will infer huge privacy risks (global coverage, possible transfer of the full EPC).

Current smart fridge has various issues. This paper proposed to address the issue of price and the issue of maturity of the smart fridge technology by introducing the use of high frequency RFID system; which is significantly cheaper than the RFID system used so far for designing the smart fridge.

\section{Proposed RFID Fridge}

The RFID Smart Fridge is an automated inventory management system allowing users to manage their refrigerated stock in real time. The RFID Smart Fridge tracks all transactions and can provide alerts configured to notify users of critical operations, for example that fridge temperature has exceeded permitted maximum, a certain product expires in few days, or that volume of a product stands below a minimum par level. In addition, a temperature profile for every product is stored from the time of entry to the time of leaving the Smart Fridge. Authorized personnel with the right access credentials only, can access the RFID Smart Fridge making the stock very secure. All data can be viewed through the Internet via mobile terminal.

Online shopping is automatically activated whenever the Smart Fridge is running out of stock. A confirmation alert is sent to the household. The fridge proceeds to execute online replenishment when the user sends back a favorable permission, while the fridge drops the command when the user gives a negative feedback. It is important to note that the inventory of online shops is stored in a database directly connected to the fridge.

\subsection{Design Architecture}

This novel RFID fridge design architecture consists of a modified fridge that includes a RFID scanner and Wi-Fi device (hardware), a wireless communication protocol (wireless communications), and online software, which receives, stores and analyzes fridge content. It is important to point out that this design is different from the existing prototypes as it uses a high frequency reader that has undergone further technical improvements to allow water permeability and minimal interference in the presence of metallic objects. Albert Lozano-Nieto (2011) focuses on the design fundamentals and highlights the technical aspects of RFID business applications.

Figure 1 illustrates our design proposal.



Figure 1. HF RFID enabled fridge 


\subsection{Requirements: RFID 13.56 MHz - High Frequency Systems}

Based on an in-depth comparative study, this research proposes a novel RFID fridge design architecture, built out of certain selected features. This research selects several standard and adaptive features to support higher read rates based on an improved RFID reader and tag operating in high frequency band. High Frequency RFID or $13.56 \mathrm{MHz}$ RFID has been utilized in a wide variety of applications where a read distance of up to 1 meter and a high security level was required. High Frequency RFID is also the least regulated and standardized bandwidth globally.

Although signals in the HF range are more affected by metal and have more attenuation when traveling through water, the transponders used for HF systems can be manufactured at lower cost than transponders operating in LF range. This is due to the fact that the antenna for HF systems can be made smaller, as shown in Figure 2. Furthermore, the need for less conductive material to construct the antennas results in a relatively low overall cost. Finally, global uniformity across the world in the frequency of $13.56 \mathrm{MHz}$ makes HF systems capable of operating in many countries.

Considering all the above facts, $13.56 \mathrm{MHz}$ promises to be an ideal frequency of choice for livestock identification, animal implantation and other applications that deal with the presence of metal and water, if systems in this HF range can be improved to a greater penetration depth and minimal attenuation when traveling through water.

We propose a Smart Fridge that is equipped with such a RFID system. A HF RFID system is required with not only great high read rates but also with water permeability and an improved penetration depth. Since standards and regulations are critical enablers for many RFID applications, the RFID system will comply with ISO15693 protocols (Barthel, 2005). This makes it well suited for applications like access control, asset tracking, warehouse management and livestock tagging.

Figure 1-2 shows an example of such improved 13.56 MHz HF RFID tag ready for animal implantation.

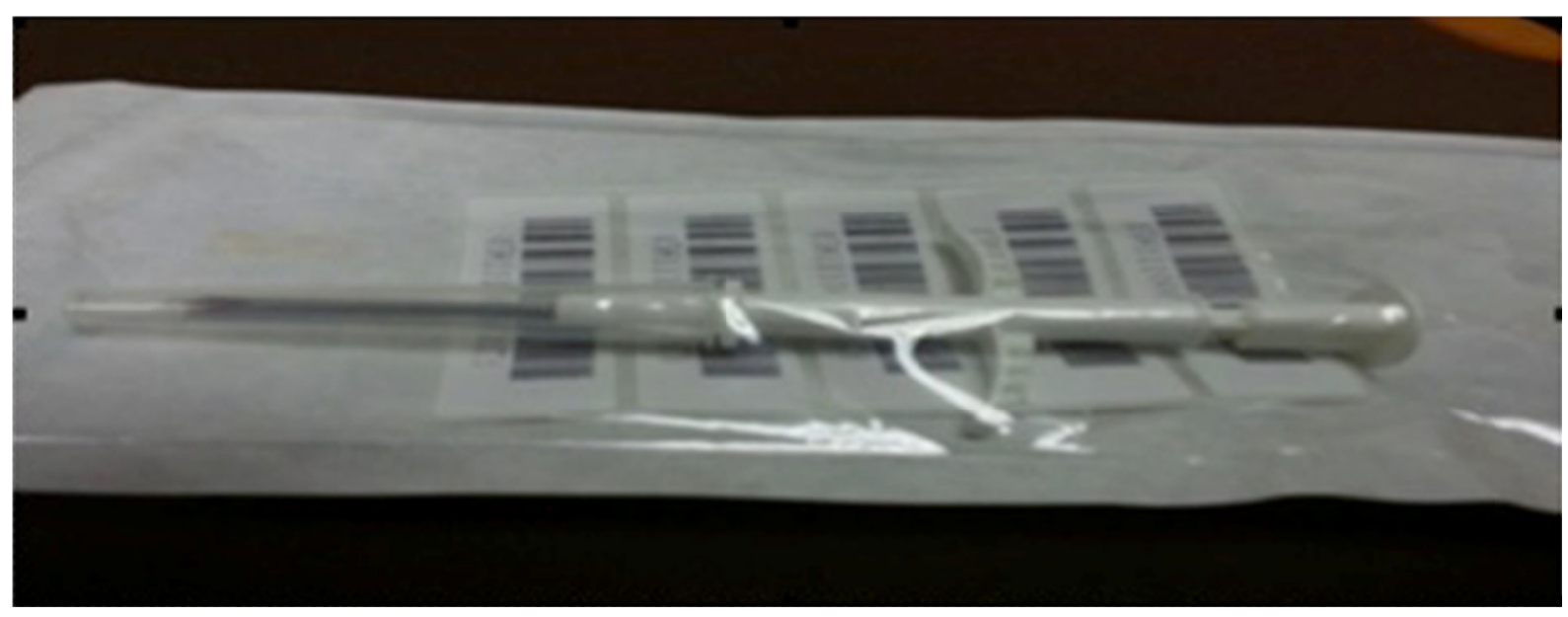

Figure 2. Improved 13.56 MHz HF RFID (water and metal resistant)

\subsection{Design Overview}

Though the smart fridge proposed in this paper does not differ from existing models when viewed from a consumer perspective, the technical approach is what makes it unique. The main advantage is the integration of HF RFID systems, which enable a better performance compared to the current design proposal. Another important fact is that since the HF RFID system design costs less than any other frequency range RFID systems, we expect this new application of RFID system to be a breakthrough in this field and to significantly lower smart fridge costs.

Figure 3 highlights the main advantage of the proposed design in this paper. 


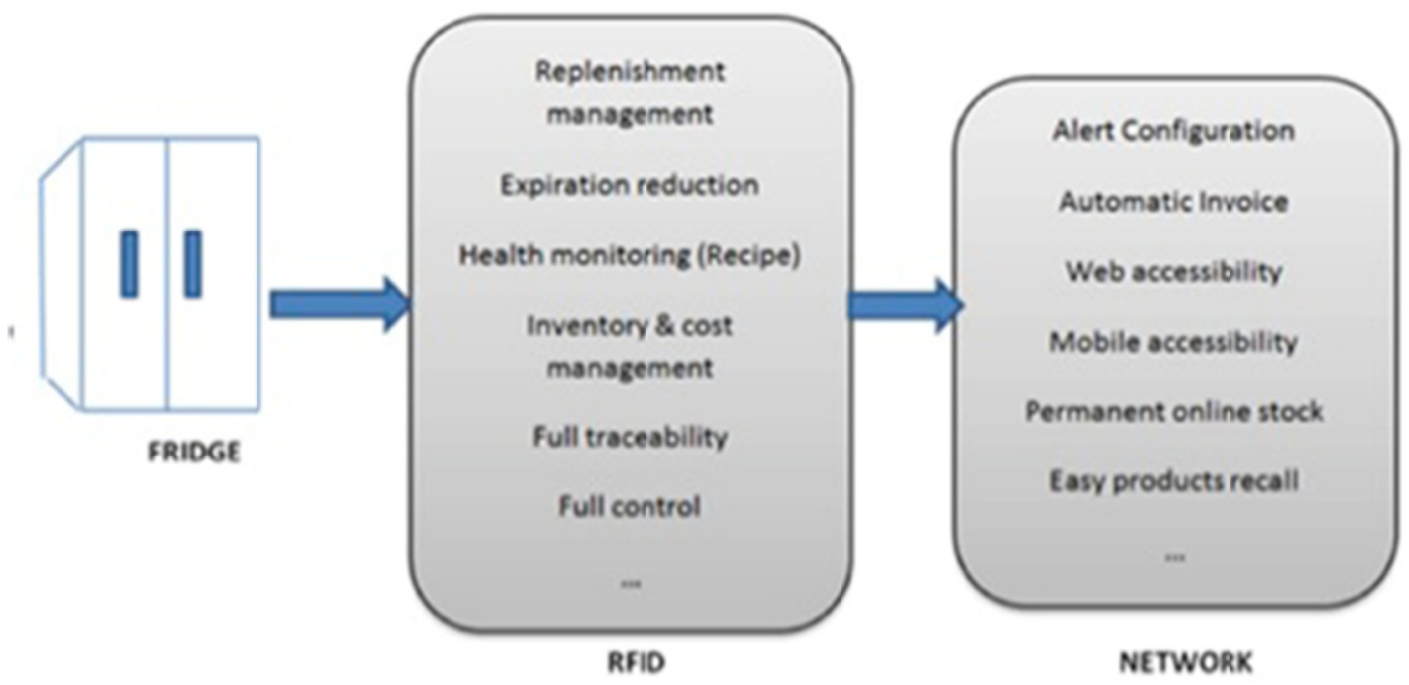

Figure 3. Networked fridge

\subsection{Characteristics}

The functionality of current refrigerators is quite limited. Whereas the traditional refrigerator struggles with food management, efficiency in shopping and cooking decision making, the RFID enabled refrigerator and its user-friendly interface offers some tremendous user-centric functions.

In busy homes, where families struggle to stay connected, we may see smart fridges serving as the hub of family activity. With calendars, weather updates, and the latest news family members can see what's on the agenda for the weekend while grabbing a quick snack Report any other analyses performed, including subgroup analyses and adjusted analyses, indicating those that were pre-specified and those that were exploratory (though not necessarily in the level of detail of primary analyses). Consider putting the detailed results of these analyses on the supplemental online archive. Discuss the implications, if any, of the ancillary analyses for statistical error rates.

\subsubsection{Inventory}

The RFID-enabled refrigerator allows customers to keep an up-to-date inventory of their refrigerated goods and have this information displayed on a LCD screen on the refrigerator door. Because each item or good is associated with a RFID tag containing information ranging from expiration date to ingredients, object name and more, the RFID enabled fridge will be able to identify items as they are taken in and out of the unit, hence eliminating food waste through constant monitoring.

\subsubsection{Recipe Suggestion with Regards to Health Conditions}

The smart fridge technology seeks to bring ease in user daily life by making the kitchen experience more enjoyable. The lack of cooking decision making encountered in the use of traditional refrigerator is addressed by providing user with a recipe generation feature. A recipe is suggested as soon as an item is being taken in or out of the unit. The more items the user takes, the larger becomes the recipe as more and more ingredients become available.

\subsubsection{Automated Replenishment}

The complete automation of the fridge unit is expressed by its ability to generate a shopping list and automatically order replenishment. The RFID refrigerator orders items online based on user consumption habits. Building on the proliferation of online services, e-commerce and home delivery services existing nowadays, the lifestyle of the user in the kitchen can be greatly improved. How would it feel to have your fridge order anything you need while you fulfill your daily task, or watch your TV and have food delivered at your doorstep, leaving the entire kitchen managed through your fridge.

\subsubsection{Interaction}

Constant human machine interaction is a key function of the smart fridge's general design. Prior to any replenishment order, a communication takes place between the RFID fridge and its user via an inbuilt Short Message Service. The user is sent a notification via his mobile device or other selected terminal whenever 
chosen stock has run out. A command confirmation by the user enables the fridge to order replenishments while a denial response orders the fridge to drop the shopping as one can decide to not purchase the same items if one's taste has changed or for any other reason.

Other types of interactions are:

- Alerts - To combat food waste and food loss, an alert message is provided on food expiration date, both before and after the period. This encourages the user to fully use all items in the fridge prior to their expiration date.

- Message Board - While a kitchen is an important environment for family gathering and care, it can also serve as a node of communication between family members. Through the smart fridge's message board, one can leave important notes to one another. A mother cooking breakfast for her child would, for example, like to know the weather so she can dress her children accordingly.

\section{Conclusion}

The features for the smart fridge proposed in this paper are similar to existing smart fridge capabilities. However, the main advantage of this design proposal resides in the innovative RFID system integrated into the fridge. The improved HF RFID system makes it possible to read multiple tagged items at the same time. Furthermore, after enhancing the penetration depth and water permeability of the RFID system, the performance of the fridge is made greater than the current proposed designs. Also, it is important to point out that the design of HF RFID system is far less expensive than other frequency ranges adopted by other designs.

In our further work, we will design the proposed smart fridge and address the security and privacy concerns that currently place a brake on the availability of the smart fridge on the market.

\section{References}

Albert, L. N. (2011). RFID design fundamentals and applications.

Amal, G. (2006). RFID Toys Cool Projects for Home, Office, and Entertainment. Wiley Publishing, Inc.

Brad, G., Alfonso, G., \& Raj. (2006). Radio Frequency Identification in Hospitals.

Chan, M., Esteve, D., Escriba, C., \& Campo, E. (2008). Review of smart homes-Present state and future challenges. Computer Methods and Programs in Biomedicine, 91(1), 55-81. http://dx.doi.org/10.1016/j.cmpb.2008.02.001

Eric, W., Nishit, R., \& Hiran, P. (2011). RFID Refrigerator Organizer. Wireless Sensor Capstone Design.

Jacqueline, B., Kristy, S., \& Ryan, R. V. (2005). Fridgets: digital refrigerator magnets. Extended Abstracts Proceedings of the 2005 Conference on Human. http://doi.acm.org/10.1145/1056808.1057099

Katherine, A. (2013). Total Surveillance. Retrieved from http://www.motherjones.com

LG. (2013). Smart Thin $Q^{\mathrm{TM}} \quad$ Refrigerator. Retrieved from http://www.lg.com/us/refrigerators/lg-LFX31995ST-french-3-door-refrigerator

Philipp Schoessler. (2011). Feedback fridge: tangible visualization of nutritional data with preventive effect. TEI '11 Proceedings of the fifth international conference on Tangible, embedded, and embodied interaction (pp. 441-442). http://dx.doi.org/10.1145/1935701.1935821

Suhuai, L., Jesse, S. J., \& Jiaming, L. (2009). A Smart Fridge with Ability to Enhance Health and Enable Better Nutrition. International Journal of Multimedia and Ubiquitous Engineering, 4(2), 39-44. http://dx.doi.org/10.1109/UMC.2008.17

Traub, K. (2005). The EPCglobal Architecture Framework, Final Edition. Retrieved from www.epcglobalinc.org/standards/ 